Research Report

\title{
Brain potentials during mental arithmetic: effects of extensive practice and problem difficulty
}

\author{
Paul Pauli a,*, Werner Lutzenberger "a Harald Rau a, Niels Birbaumer ${ }^{\mathrm{a}, \mathrm{c}}$, \\ Timothy C. Rickard ${ }^{b}$, Rita A. Yaroush ${ }^{b}$, Lyle E. Bourne Jr. ${ }^{b}$ \\ "Unicersity of Tübingen, Institute of Medical Psychology and Behatioral Neurobiology, Gartenstrasse 29, D-72074 Tübingen, FRG \\ ${ }^{b}$ Unicersity of Colorado, Department of Psychology, Institute of Cognitite Science, Roulder, Colorado, USA \\ ${ }^{c}$ Unitersitá degli Studi, Padora, Italy
}

Accepted 22 March 1994

\begin{abstract}
Recent behavioral investigations indicate that the processes underlying mental arithmetic change systematically with practice from deliberate, conscious calculation to automatic, direct retrieval of answers from memory [Bourne, L.E.Jr. and Rickard, T.C., Mental calculation: The development of a cognitice skill, Paper presented at the Interamerican Congress of Psychology, San Jose, Costi1 Rica, 1991; Psychol. Ret., 95 (1988) 492-527]. Results reviewed by Moscovitch and Winocur [In: The handbook of aging and cognition, Erlbaum, Hillsdale, NJ, 1992, pp. 315-372] suggest that consciously controlled processes are more dependent on frontal lobe function than are automatic processes. It is appropriate, therefore to determine whether transitions in the locus of primary brain activity occur with practice on mental calculation. In this experiment, we examine the relationship between charicteristics of event-related brain potentials (ERPs) and mental arithmetic. Single-digit mental multiplication problems varying in difficulty (problem size) were used, and subjects were trained on these problems for four sessions. Problem-size and practice effects were reliably found in behavioral measures (RT). The ERP was characterized by a pronounced late positivity after task presentation followed by a slow wave, and a negativity during response indication. These components responded differentially to the practice and problem-size manipulations. Practice mainly affected topography of the amplitude of positivity and offset latency of slow wave, and problem-size mainly offset latency of slow wave and pre-response negativity. Fronto-central positivity diminished from session to session, and the focus of positivity centered finally at centro-parietal regions. This finding suggests that frontal lobe processing is necessary as long as task performance is not automatized, while automatized arithmetic processing requires parietal brain activity only. The pre-response negativity observed in the first session and during more difficult tasks is assumed to reflect excitatory preparatory processes, which could be associated with activation of calculation strategics.
\end{abstract}

Key words: Mental arithmetic; Event-related potential; Practice; Automatization; Problem size

\section{Introduction}

Mathematics and arithmetic form a prototypical cognitive system, defined by (largely) specifiable rules which are almost universally understood in modern society. System or domain knowledge and the skills to use that knowledge are acquired relatively early in life (although lcarning and improvement clearly can continue throughout a lifetime), and are used in grossly similar ways by all adults. Both cognitive psychologists

\footnotetext{
* Corresponding author. Fax: (49) 7071-295956. E-mail: pauli@comserv1.zdv.uni-tuebingen.dbp.de
}

and psychophysiologists have employed mental arithmetic problems as a paradigm to study higher order mental processes.

Some important facts are beginning to emerge from behavioral studies of arithmetic and mathematical processes (see special issue of Cognition, 1992). Much of this empirical work has used a mental arithmetic task, in which subjects are given single-digit problems such as $8+3=$ ? or $6 \times 4=$ ? and are scored primarily according to speed of answer production. These are relatively simple problems for adult subjects, yet practice reveals significant performance speed-up, following the power law [19,25]. Another result, reliably observed across a number of experiments, is the prob- 
lem-size effect [6,36,38]. Roughly speaking, the larger the product of any multiplication operation, the more time it takes to produce a correct answer. The problem size effect decreases with practice, but has not been eliminated even in experiments which have provided up to 12 sessions of sustained practice on a subset of single-digit mental multiplication problems [13].

Theoretical interpretations of mental arithmetic emphasize one or both of two fundamental processes, fact retrieval and rapid calculation. On the one hand highly skilled mental multiplication appears primarily to depend on retrieval from an associative network of arithmetic facts. This conclusion is documented by interference and priming effects in both normal and braindamaged subjects, which indicate that answer production is primarily a matter of retrieving numeric facts from memory and that fact retrieval reflects associative network processes in which multiple problem and answer representations are activated during every retrieval attempt $[8,28,29,37]$. On the other hand, some studies, especially those using strategy probing techniques $[2,5,36]$ suggest that access to stored answers is not the only problem solving mechanism available. Under some circumstances, subjects calculate or derive a correct answer by mediating strategies, such as repeated addition $(7 \times 3=7+7+7)$, anchor and adjust $(8 \times 6=8 \times 5+6)$ or the like.

The theoretical framework of McCloskey, Caramazza and Basili [22] incorporates both a retrieval and a calculational route to answer production. In this system, arithmetic problems are interpreted within an encoding subsystem that translates the perceptual characteristics of a problem, as presented, into an abstract format suitable for processing within a second subsystem that incorporates both calculational strategies and direct access to numerical facts. Direct access is generally a faster route to arithmetic facts but weak associative connections and interference processes can create circumstances in which answers are produced through one or another calculational procedure. In either case, the answer, once attained in abstract form, must be decoded to produce a response program capable of execution within the parameters of the task.

As an empirical understanding of mental arithmetic emerges, it is natural to ask whether there is neurophysiological evidence for putative cognitive components of the task and about possible systematic relationships between these brain activities and variables, like problem size and practice, that are known to affect speed and accuracy of performance. For example, recent results $[5,27]$ imply that part of the speed-up in response times with practice is attributable to a transition from algorithmic answer calculation on early trials to direct access to answers in a fact network for all problems on later trials. The basis of performance changes with practice from deliberate, conscious effort to generate an answer to automatic, effortless answer retrieval. Results reviewed by Moscovitch and Winocur [24] suggest that consciously controlled processes are more dependent on frontal lobe function than are automatic processes. Therefore, it is entirely reasonable to expect that practice in mental arithmetic will lead to a detectable transition in primary locus of brain activity.

The EEG offers a way to monitor brain activity in real time and thereby to track any transitions that parallel changes in behavior. Moreover, event-related potentials (ERPs) are signs of specific stages of information processing $[4,10]$. There is some evidence of an association between slow brain potentials and the task demands of mental arithmetic. Onc finding is that more difficult tasks are associated with more positive amplitude levels $[9,33]$. Further, mental arithmetic tasks seem to be associated with a relative positivity over the frontal cortex $[3,32,33,34,35]$. The interpretation of this frontal positivity is somewhat controversial. Rösler and Heil [32] related the frontal positivity to the mental operation of division and Ruchkin et al. [35] to the allocation of resources and/or to the coordination of processing states.

The present study uses a somewhat different approach to seek a further refinement of the relationship between performance in mental arithmetic and characteristics of the ERPs. So far, all studies varied task difficulty by using different kinds of mental operations, for example, comparing performance on addition versus multiplication problems $[9,32,34,35]$. We used only one kind of operation (single digit multiplication) and evaluated the effect of two independent variables, problem-size and practice, on FRPs. Two main hypothesis were tested: (1) The problem-size of the task is directly related to the amount of positivity in the ERP. (2) Because practice makes direct retrieval more and calculation strategies less likely, it should lead to a focusing of brain activity. In particular, we expect a reduction of frontal brain activity with practice. In contrast, temporo-centro-parietal regions are functionally necessary for automated mental arithmetic, implying that activity in this region will remain relatively stable with practice.

\section{Materials and methods}

\subsection{Subjects}

Fifteen right-handed subjects ( 12 male, 3 female) participated in the experiment. Handedness was verified with the Edinburgh Handedness Inventory [26]. One man was excluded because he attended only the first session. The final data pool included 14 subjects aged 21 to 33 . All were highly educated, 12 were university students, one a computer technican, and one a nurse. One female subject missed the fourth practice session. One woman was excluded from EEG analysis 
due to frequent EEG artefacts. Subjects were paid DM 60 for attending all sessions.

\section{2. irocedure and design}

Subjects sat in a reclining chair in front of a computer screen (distince about $2 \mathrm{~m}$ ). The screen continously displayed numbers from ") to 9 (size of $2 \mathrm{~cm} \times 1 \mathrm{~cm}$ ) arranged in a circle (radius $=9 \mathrm{~cm}$ ), wher 0 was at top and the numbers increased in a clockwise order. An " was displayed in the middle of the circle, meaning 'ready' (in Gerraan 'fertig'). $\wedge$ small table was mounted in front of the subjects. so th ast a computer mouse could easily be moved on a mouse-pad with the right hand. A cursor on the computer screen (in form of a hanc with a pointing index finger) could be controlled with the mouse. When the cursor was positioned at the ' $f$ ', the multiplication problem appeared after some delay directly above the ' $f$ ' in the following format: ' $7 \times 2=\ldots$. The time delay between pointer on the ' $f$ ' and task presentation varied randomly between 0.5 and $1.5 \mathrm{~s}$ in orde to reduce subjects' expectancies about task timing. Subjects could indicate the product by moving the cursor to the first and then to the second digit of the answer (e.g. for the problem ' $7 \times 2=$-' subjects had to move first to ' 1 ' and then to '4'). Wrong answers were indic ted by changing the pointer into an open hand, and subjects could only continue when they indicated the correct answer.

Only single-digit multiplication problems were used, with square problems (e.g. $4 \times 4$ ) and problems with one digit answers excluded. The remaining set of 52 single-digit multiplication problems was divided into 26 commuted pairs, with the two problems in each pair differing only in operand order (e.g. $6 \times 5$ and $5 \times 6$ ). A set of 26 problems (one from each) was constructed, including 13 problems selected at random with the operands in ascending order (e.g. $4 \times 5)$ and the remaining 13 problems with the operands in descending order (e.g. $7 \times 4$ ). Each subject received 20 blocks of these 26 problems per session. Problem order within blocks was determined pseuilorandomly for each subject.

Subjects were tested during four sessions within 2 weeks, each lasting less than $90 \mathrm{~min}$ including preparations for the physiological reco:dings. Sessions were run in the morning at 9 a.m. or $10.30 \mathrm{a} . \mathrm{m}$. Subj:cts received written instruction about the mental arithmetic task it the beginning of the first session. Instructions emphasized that they should try to solve the tasks as fast and accurately as possible, and that they should keep on trying to improve their performance throughout each session. Subjects were instructed to keep their eyes focused on the center of the screen, and to avoid unnccessary body and eye movements. They were informed that they could' have a break by delaying the cursor movement to the ' $f$ ' in the midcile of the screen. Ten practice trials were run in the first session to familiarize subjects with the task. A short version of the instructions was repeated at the beginning of each session.

\section{3. $\because E G$ recording}

The EEG was recorded from 17 sites using a commercially available electrode cap with tin electrodes placed according to the international 10-20 system. $\mathrm{Ag} / \mathrm{AgCl}$ electrodes for reference were placed on the mastoids, and for vertical and horizontal eye movemen's above and below the right eye. All impedances were kept belox $5 \mathrm{k} \Omega$. All channels were recorded with a $\mathrm{Cz}$ reference (bandwidt $10.0796-35 \mathrm{~Hz}$, sampling rate $100 \mathrm{~Hz}$ ) and converted off-line relative to linked ears. Trials with large eye movements $(>100 \mu \mathrm{V})$ were excluded. EOG correction was applied by means of a constant fraction method [12]. A DEC PDP $11 / 73$ computer controlled stimulus timing, digitized physiological recordings and recorded subjects reacion times. EEG and EOG was digitalized for $4 \mathrm{~s}$, starting $0.5 \mathrm{~s}$ before task presentation.

\subsection{Data reduction and statistical analysis}

Three reaction times were measured: $\mathrm{RT} 0$ reflects the time between the presentation of the task and the beginning of the cursor movement, RT1 the time between the task presentation and the indication of the first and RT2 of the second answer digit.

The arithmetic problems were divided into three difficulty categories, depending on the product (easy: 10-21; moderate: 24-40); difficult: 42-72). The distance from the ' $\mathrm{f}$ ' in the middle of the circle to the first answer digit was the same for all tasks. However, this was not true for the distances between first and second answer digits (e.g. the distance for 42 is shorter than for 27). In order to contuol for this effect, time to move the cursor from the first to the second response digit was calculated for each problem (RT2-RT1). Sixteen problems. which were comparable in the RT2 - RT1 differences, were chosen for further analysis (easy: $3 \times 4,6 \times 2,2 \times 7,2 \times 9,6 \times 3,5 \times 4$; moderate: $8 \times 3,4 \times 6,4 \times 8,7 \times 5,9 \times 4$; difficult: $5 \times 9,6 \times 8,6 \times 9,8 \times 7$. $7 \times 9)$.

The distribution of RT revealed two kind of errors. In one case, subjects started to move the cursor immediately after the presentation of the task without having the solution in mind. This is reflected in RT0 faster than $100 \mathrm{~ms}$. In the other case, subjects did not indicate the result within the registration period of $4 \mathrm{~s}$. Trials with RT0 faster than $100 \mathrm{~ms}$ and trials with RT2 slower than $4 \mathrm{~s}$ were excluded from all further analysis. The behavioral data (RT) were analyzed with two-factorial repeated measurement ANOVAs with the within factors PRACTICE (session 1 to 4) and DIFFICULTY (easy, moderate, difficult). Dependent variables were RT0, RT1 and RT2.

Two types of ERP averages were computed separately for subjects, 9 electrode sites (F3, Fz, F4, C3, Cz, C4, P3, Pz, P4), four practice sessions (S1 to $\mathrm{S} 4$ ), and three levels of difficulty (easy, moderate, difficult): (1) Stimulus-synchronized and (2) response-synchronized averages. Response-synchronized averages were confined to trials with RT0 greater than $500 \mathrm{~ms}$ and RT2 less than $3 \mathrm{~s}$ (this was done to have a registration interval of at least $500 \mathrm{~ms}$ pre- and post-response indication). Repeated measurement ANOVAs with the within factors PRACTICE, DIFFICULTY and ELECTRODES were conducted to assess significance of the experimental manipulations. Significant overall effects in the ANOVAs were further analyzed by post-hoc means comparisons. When appropriate, the Greenhouse-Geisser epsilon (G-G) was used to correct degrees of freedom, and only corrected significance levels are reported.

The following dependent measures were measured separately for subjects, electrodes, practice sessions and levels of difficulty: (1) Amplitude of positivity of the stimulus-synchronized average: Average peak amplitude $( \pm 50 \mathrm{~ms})$ around the most positive peak within the first $500 \mathrm{~ms}$ after task presentation; (2) Offset latency of slow wave of the stimulus-synchronized average (This second parameter refers to the time difference between task presentation and end of the positive slow wave. The end of the slow wave is defined as the first data point with a negative value relative to the baseline); and (3) Pre-response amplitude of the response-synchronized average: Mean amplitude over $100 \mathrm{~ms}$ immediatly before RT0.

\section{Results}

\subsection{Behavioral data}

For all three reaction times (RT0, RT1, RT2) ANOVAs revealed highly significant PRACTICE and DIFFICULTY main effects and PRACTICE by DIFFICULTY interactions (Table 1). The more difficult 
Table 1

ANOVAs with repeated measures on four practice sessions $(\mathrm{P})$ and three problem difficulty levels (D) for the three behavioral measures, RT0 (beginning of the cursor movement), RT1 (indication of the first answer digit), and RT2 (indication of the second answer digit)

\begin{tabular}{|c|c|c|c|}
\hline $\mathrm{df}$ & RT0 & RT1 & RT2 \\
\hline & $\Gamma \quad$ G-G $P$ & $F \quad$ G-G $P$ & $F \quad$ G-G $P$ \\
\hline
\end{tabular}

Practice $3,3927.70 .56<0.0031 .70 .68<0.0026 .70 .61<0.00$ Difficulty $2,2646.30 .58<0.0041 .60 .64<0.0039 .70 .61<0.00$ $\mathbf{P} \times \mathbf{D} \quad 6,78 \quad 12.00 .41<0.0011 .6 \quad 0.44<0.00 \quad 9.30 .48<0.00$

$\mathrm{G}-\mathrm{G}$ refers to Greenhouse-Gneisser epsilon.

the task, the greater the $\mathrm{RT}$ reduction with practice. This observation is verified by post-hoc means comparisons, which reveal that RT0 for difficulty tasks significantly decreased from session to session (S1 vs S2, $P<0.0001 ; \mathrm{S} 2$ vs. S3, $P=0.009$; S3 vs. S4, $P=0.02$ ), whereas this is not true for easy tasks (S1 vs. S2, $P=0.005$; S2 vs. S3, n.s.; S3 vs. S4, n.s.). However, difficult tasks still yield longer RT0s than easy tasks even after four practice sessions (S4-easy vs S4-difficult, $P<0.0001$ ). Similar effects were found for RT1 and RT2, but in order to avoid redundancy will not be explicitly presented. Consistent with the power law of practice, log RT0 decreases linearly with log session (Fig. 1).

\section{2. $E E G$}

Stimulus synchronized ERPs (grand averages over all electrodes) differentiated for task difficulties and practice sessions are presented in Fig. 2. Task difficulty seems to be reflected mainly in the offset latency of slow wave, while practice affects both the amplitude of positivity and the offset latency of slow wave. Negativity peaked clearly after response initiation (RT0) under all conditions, however, the pre-response amplitude

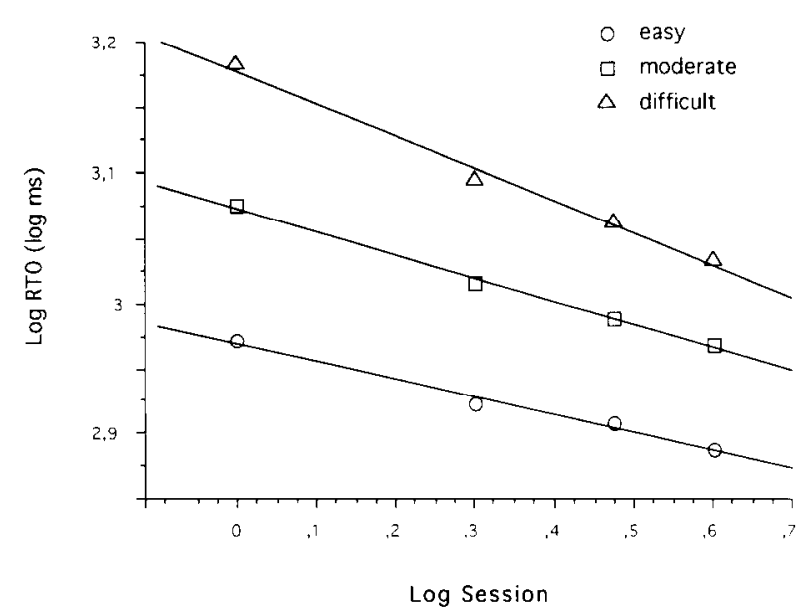

Fig. 1. Log reaction times plotted as a function of log practice session and problem difficulty $(\mathrm{RT} 0=$ beginning of the cursor movement).

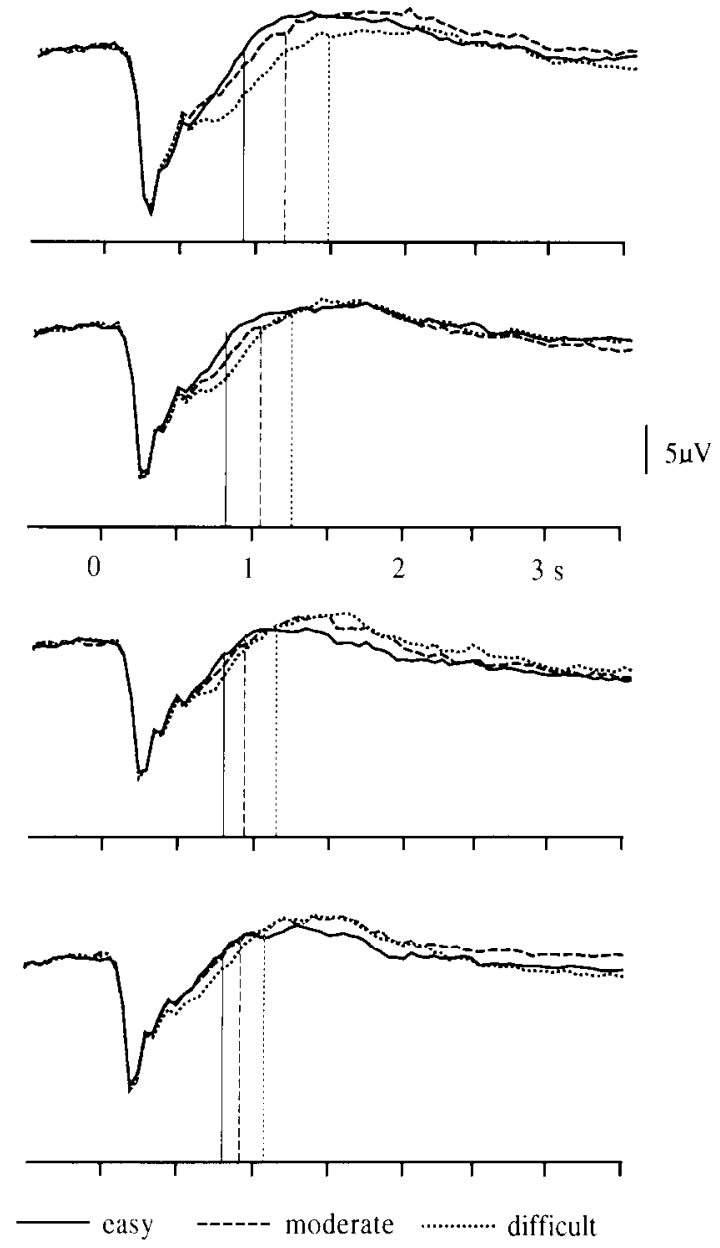

Fig. 2. Stimulus-synchronized grand averages (over all electrodes) differentiated according to four practice sessions (presented from the top to the bottom as the first to the fourth session, respectively) and three problem difficulty levels. Solid lines: easy problems; dashed lines: moderate problems; dotted lines: difficult problems. Problem presentation was at second zero. Mean reaction times (RT0) are indicated by vertical lines. Waves are plotted with negative amplitude up.

seems to be more negative as a function of task difficulty.

Amplitude of positivity. The ANOVA revealed significant PRACTICE $\left(F_{3,36}=19.3, \quad \mathrm{G}-\mathrm{G}=0.72, \quad P<\right.$ $0.0001)$, ELECTRODES $\left(F_{8,96}=18.3, \mathrm{G}-\mathrm{G}=0.24, P\right.$ $<0.0001)$, and PRACTICE by ELECTRODES $\left(F_{24.288}\right.$ $=3.6, \mathrm{G}-\mathrm{G}=0.24, P=0.004)$ effects. In session 1 , positivity is higher at $\mathrm{Cz}$ than at $\mathrm{Pz}(P=0.005)$, and higher at $\mathrm{Pz}$ compared to $\mathrm{Fz}(P=0.0003)$, as shown in Fig. 3. Practice then leads to a clear reduction in positivity at frontal and central recording sites from Session 1 to 2 and from Session 2 to 3 but not from Session 3 to 4 (Fz: S1 vs S2, $P=0.01 ; \mathrm{S} 2$ vs S3, $P=0.005$; S3 vs S4, n.s.; Cz: S1 vs S2, $P=0.004$; S2 vs $\mathrm{S} 3, P=0.01$; 33 vs $\mathrm{S} 4$, n.s.). Similar session to session changes were not significant for parietal electrodes (Pz: S1 vs S2, n.s.; S2 vs S3, n.s., S3 vs S4, n.s.), and in 


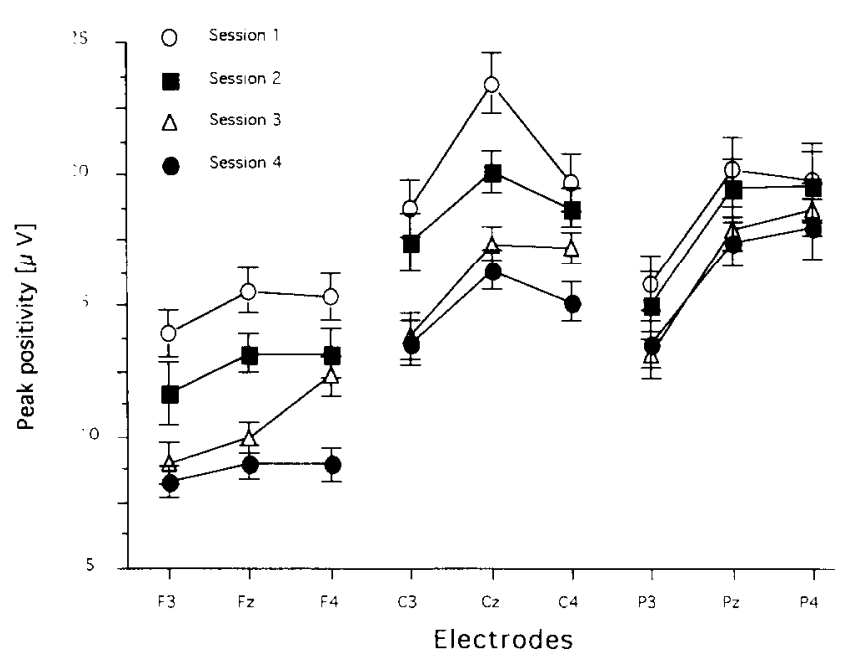

Fig. 3. Mean peak positivity $( \pm$ S.E.M. $)$ as a function of practice sessions and electrode locations.

Session $4 \mathrm{Cz}$ and $\mathrm{Pz}$ positivity was about the same and in both cases significantly higher than at $\mathrm{Fz}(\mathrm{Cz}$ vs $\mathrm{Fz}$, $P=0.0001 ; \mathrm{Pz}$ vs Fz, $P=0.0001)$. In addition, for parietal electrodes the positivitity is less pronounced on left compared to right recording sites (S1: P3 vs P4, $P=0.001 ; \mathrm{S} 4: \mathrm{P} 3$ vs $\mathrm{P} 4, P=0.0005)$. Comparable lateralization effects were not found for frontal or central sites (S1: F3 vs F4, n.s.; C3 vs C4, n.s.; S4: F3 vs F4, n.s.; C3 vs ( 4 , n.s.). No main or interaction effects involving the factor DIFFICULTY reached significance.

Offset latency of slow wave. The offset latency of slow wave is influenced by DIFFICULTY $\left(F_{2.24}=5.4\right.$,

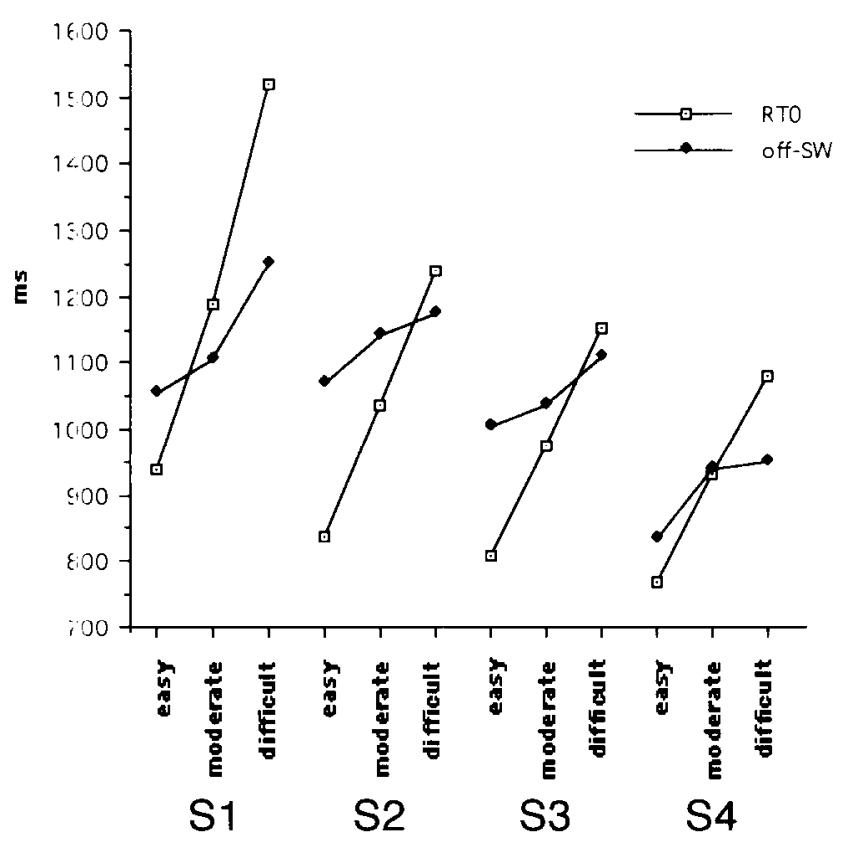

Fig. 4. Mean offset latency of slow wave (off-SW) and reaction time (RT0) for four practice sessions and three problem difficulty levels.
$\mathrm{G}-\mathrm{G}=0.93, P=0.01)$ and by PRACTICE $\left(F_{336}=4.6\right.$, $\mathrm{G}-\mathrm{G}=0.65, P=0.02)$. No other main or interaction effect and no effect involving ELECTRODES reached significance. Task difficulty leads to an increase (easy: $991.6 \mathrm{~ms} \pm 587.1$; moderate: $1055.6 \pm 612.1$; difficult: $1121.4 \pm 611.8$ ), and practice to a decrease (S1: 1136.1 $\pm 587.6 ;$ S2: $1129.1 \pm 627.2 ;$ S3: $1050.4 \pm 604.8 ;$ S4: $909.1 \pm 578.1$ ) in the offset latency of slow wave (Fig. 4). Post-hoc comparisons show that, regarding difficulty, only the difference between easy and difficult tasks reaches significance $(P=0.004)$. Regarding practice, there was no significant overall change from one session to the other, but the difference between Session 1 and 4 is highly significant $(P=0.008)$.

Pre-response amplitude (response-synchronized). Fig. 5 depicts the response-synchronized ERPs (grand averages over all clcetrodes) differentiated for the three difficulty levels and the four practice sessions. The ANOVA revealed significant DIFFICULTY $\left(F_{2.24}=\right.$ 3.5, G-G $=0.91, \quad P=0.05$ ) and DIFFICULTY by ELECTRODES $\left(F_{16,192}=3.3\right.$, G-G $=0.24, \quad P=0.02$; Fig. 6) effects, and a marginal significant PRACTICE $\left(F_{3,36}=3.3, \quad \mathrm{G}-\mathrm{G}=0.63, \quad P=0.057\right) \quad$ effect. Pre-response amplitudes were more negative for difficult and moderate compared to easy tasks at parietal recording
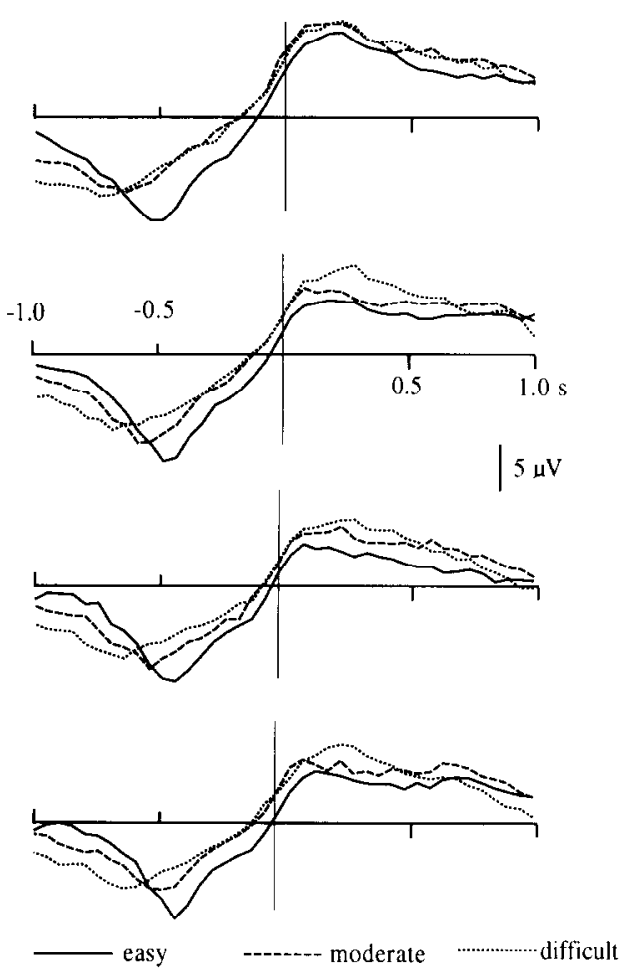

Fig. 5. Response-synchronized grand averages (over all electrodes) differentiated according to four practice sessions (presented from the top to the bottom as the first to the fourth session, respectively) and three problem difficulty levels. Solid lines: easy problems; dashed lines: moderate problems; dotted lines: difficult problems. Response initation (RT0) was at second zero. Waves are plotted with negative: amplitude up. 
sites (Pz: easy vs difficult, $P=0.05$; easy vs moderate, $P=0.005)$, whereas frontal $(\mathrm{Fz})$ and central $(\mathrm{Cz})$ preresponse amplitudes did not differ for different task difficulties (Fig. 6). In addition, during difficult tasks pre-response amplitude was more negative at left compared to right frontal and central electrode sites (difficult tasks: F3 vs F4, $P=0.03$; 33 vs C4, $P=0.004 ; \mathrm{P} 3$ vs $\mathrm{P} 4$, n.s.). Comparable lateralization effects were not found for moderate and easy tasks. The marginal significant PRACTICE effect can be traced back to a more negative pre-response amplitude in the first session compared to all other sessions (S1: $-3.5 \mu \mathrm{V}$ $\pm 8.1 ; \mathrm{S} 2:-1.4 \pm 8.6 ; \mathrm{S} 3: 1.1 \pm 8.7 ; \mathrm{S} 4: 1.6 \pm 7.7 ; \mathrm{S} 1$ vs S2, $P=0.03$; S1 vs S3, $P=0.02$; S1 vs S4, $P=0.05)$.

\subsection{Correlation between EEG and behatioral data}

RT0 is a behavioral and offset latency of slow wave (off-SW) is a neurophysiological parameter for processing time. Fig. 4 depicts the relation between these variables differentiated for problem difficulty and practice. Two effects are visible: First, response indication (RT0) for easy tasks takes place during positivity and for difficult tasks during negativity. Second, RT0 and offset latency of slow wave become increasingly more similar with practice. An ANOVA with the within factors PRACTICE, DIFFICULTY and PARAMETERS (RT0 vs offset latency of slow wave) confirms this. There is no overall difference between the PARAMETERS, but there is a significant PARAMETER by DIFFICULTY $\left(F_{2.24}=14.2, \quad \mathrm{G}-\mathrm{G}=0.95, \quad P=\right.$ $0.0001)$ and a marginally significant PARAMETER by PRACTICE interaction $\left(F_{3.36}=2.7, \mathrm{G}-\mathrm{G}=0.80 . P=\right.$ 0.07). Post-hoc mean comparisons reveal that for easy tasks RT0 was significantly shorter than offset latency of slow wave $(P=0.001)$, while for difficult tasks the opposite was true $(P=0.007)$. Regarding practice, RT0 was significant larger than offset latency of slow wave in the first session $(P=0.05)$. No significant differences were found for the other sessions.

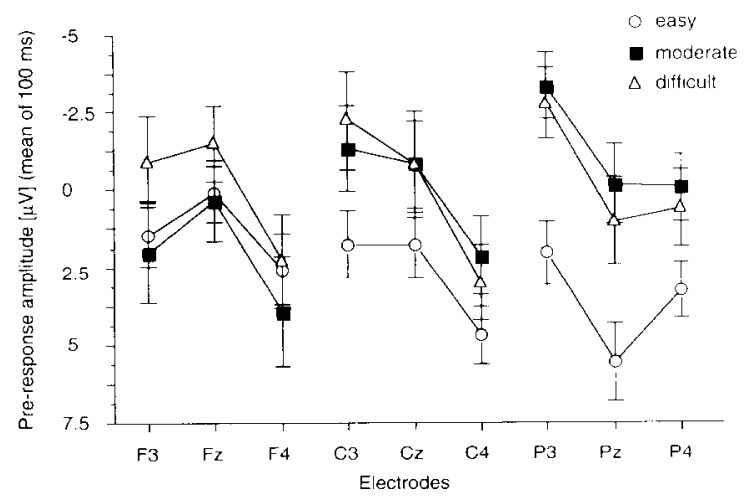

Fig. 6. Mean pre-response amplitude $( \pm \mathrm{SEM})$ according to three problem difficulty levels and nine electrode locations.

\section{Discussion}

Response time speeds up significantly across practice sessions, and this speed-up follows the power law (see Fig. 1). Practice also leads to an attenuation of the problem-size effect, although, the effect is still reliable in the fourth session. These results are in agreement with Fendrich, et al. [13], who used similar problems but a different response indication procedure. Still, the mean reaction times in their study (time until subjects typed the first answer digit on a numeric keypad) are comparable with our RT0. The close comparability between these studies underlines the suceess of adopting Fendrich, et al.'s mental arithmetic paradigm to an EEG study.

The ERP is characterized by a pronounced late positivity followed by a slow wave, and a negativity slightly before and during response. The late positivity peaks at $300 \mathrm{~ms}$ and seems to be a 'P300-like' component. However, we are reluctant to label it a P300, because if does not fulfill the criteria of a classical P300 [11] (i.e. no clear parietal maximum). This late positive component is followed by a slow wave lasting from about 500 to $1000 \mathrm{~ms}$.

The observed components are related, but differently to practice and problem difficulty. Practice mainly affected the offset latency of slow wave and the topography of positivity. In the first session, positivity was focused at $\mathrm{Cz}(22 \mu \mathrm{V})$ and $\mathrm{Pz}(20 \mu \mathrm{V})$, but also reached a considerable peak at $F_{Z}(15 \mu \mathrm{V})$. With practice, frontal and central positivity significantly decreased, while parietal positivity was unaffected. In the fourth session, then, positivity was highest at Pz. The amplitude of positivity did not vary with problem difficulty. Offset latency of slow wave was less with easy tasks and decreased with practice. Pre-response negativity was especially high in the first training session. and a higher pre-response negativity at parietal recording sites during difficult as compared to easy tasks was found. In addition, for difficult tasks, pre-response amplitude was more negative at left compared to right frontal and central recording sites.

In the first session, positivity could be observed at all electrode sites, suggesting that frontal, central, and parietal corticies were involved in the processing of the problem. With practice, frontal and central positivity substantially decreased, while parietal positivity was unaffected. Neuropsychological studics indicate that the frontal brain is involved whenever mental activity is deliberate and controlled $[15,20,24]$. Moreover, frontal brain lesions cause deficits when remembering requires the initiation and maintenance of effortful strategies of encoding and/or retrieval [21]. From this point of view, it makes sense that the more the processing of the arithmetic task becomes automatized or proceduralized, the less frontal cortical activity will be ob- 
served. Such an outcome is also consistent with the idea that frontal activity may reflect an executive function which allocates resources and/or organizes the processing stages of the task $[31,35]$. Automatized processing no longer requires deliberate resource allocation. The finding that parietal positivity did not change with practice suggests that this brain region is functionally necessary to answer retrieval, by direct access [29] rather than by conscious calculational procedure. This evidence also fits with neuropsychological data, showing that arithmetic deficits in adults, who developed high arithmetic skill prior to brain insult, tend to be associated with parietal brain lesions [15,16]. Neurophysiological studies using an irregularity index of the EEG $i$ or measures of regional blood flow which reflect cortical activation also found a increased temporocentro-parietal activity during mental arithmetic [14,31].

The interpretation of parietal positivity as a sign of answer retrieval gains support by the observation that offset latency of slow wave decreased with practice and increased with problem-size. The easier the task and the more practice is completed, the quicker the problem gets processed. However, problem-size did not effect the topography or amplitude of positivity. This could mean that easy and difficult problems activate similar cortical networks, whereby difficult problems need more processing time because of less established problem-to-answer association-pathways. Rickard and Bourne [28] recently developed an interactive activation model of mulliplication fact retrieval that is consistent with this interpretation (for related models, see $[1,7.23])$. The basic architecture assumes localist. abstract representations at three levels: (a) an element level, in which the three mathematically essential elements of the problem (e.g. 4,9, and $\mathrm{x}$ for the problem $\cdot 4 \times 9=$ ') are represented. (b) a problem level in which there is a unique node representing each problem, and (c) an answer level, in which there are nodes representing arithmetic answers. There are excitatory connections between levels. For example, the elements 4,7, and $\mathrm{x}$ have excitatory connections to the problem node $4 \times 7$. There are inhibitory connections within levels. Performance is simulated by providing activation to problem elements of the first level, and then letting this activation spread cyclically to the problem and answer levels. During the initial cycle, multiple problem and answer nodes become active. As cycling proceeds, all but one problem - answer node combination (usually the correct one) are typically inhibited. In this model, the number of candidate problem and answer nodis that become active early in processing does not vary systematically with problem difficulty. This characteristic of the model seems to map closely onto the observation of no difference in peak positivity attributable to problem difficulty. However, because associations among problem and answer nodes are as- sumed to be weaker and less coherent for difficult problems, more cycles are necessary to suppress incorrect problem and answer nodes for difficult problems, which is again consistent with the ERP data.

Pre-response negativity was found to be significantly reduced for easy compared to difficult arithmetic tasks at parietal recording sites, and pre-response negativity generally declined as training progressed. Similar results have been reported by Ruchkin et al. [35], who used addition or substraction as easy tasks and division for difficult problems. Negativity was larger for more difficult than easy problems, and this effect was more pronounced at $\mathrm{Pz}$ and $\mathrm{Cz}$ compared to $\mathrm{Fz}, \mathrm{Oz}$, and Fpz. In both studies (see also [3]) the response related slow-wave was more pronounced at left compared to right recording sites. Thus, in spite of the different behavioral paradigms, the results regarding the response related slow-wave amplitude are in good concordance.

How might the response-related negativity be interpreted behaviorally? A direct association with response production is unlikely, since responses were very similar for easy and difficult task (see also [35]) and no unequivocal relation between response indication and ERP-negativity was found (see Fig. 4). Somehow, negativity must reflect the association between production and motor programming prior to response execution. That is, the manner in which the response in prepared, which depends on what happens cognitively, seems to be the most likely concommitant of negativity in the EEG. The prior occurrence of mediating strategies might be implicated. Consider that, in the first session and during more difficult tasks, it is likely that subjects are not able to retrieve aritmetic facts from memory directly. Back-up calculation strategies are activated [2], leading to excitatory processes in parietal brain areas producing negativity in the EEG [4]. In this context, the relatively large and longlasting negativities found by Ruchkin et al. [34,35] and Rösler and Heil [32] during mental arithmetic could be attributed to their use of complicated and unusual arithmetic tasks (e.g., subjects had to divide a 3 -digit number by 7 and compute the remainder). This interpretation would also fit the observation that cortical negativity covaries with signs of conscious awareness of mental processes $[17,18,30]$. Conscious processing is more likely at the beginning of training and during difficult tasks [24].

One critical question is whether this negativity confounded the measurement of the offset latency of slow wave and thus leads us to draw misleading conclusions about the offset latency of slow wave. The data indicate that negativity was greater for difficult and moderate compared to easy tasks at parietal recording sites, and tended to be greater during the first compared to the other sessions. This could have led to a shortening of the positive slow wave under these conditions. How- 
ever, we still found that the offset latency of slow wave was increased at all recording sites for difficult tasks and in the first session. It is very unlikely that our findings are due to a confounding of the measures, since the negativity effect occurs at parietal electrodes only, and the offset latency of slow wave effect at all recording sites. Additionally, a confounding would have led to an understimation of the effects and not to misleading conclusions.

In summary, our data show that skill acquisition for simple multiplication problems through practice leads to clear behavioral changes measured by response time. Moreover, skill aquisition was reflected in systematic ERP changes. Fronto-central positivity diminished from session to session, and the focus of positivity centered finally at centro-prietal regions. Positivity was interpreted as a sign for retrieval of arithmetic facts from cortical networks. Since problem-size did not affect amplitude or localization of positivity, hut, was related to offset latency of slow wave, one could speculate that difficult and easy tasks activate the same cortical networks, and only differ in processing time. The high pre-response negativity observed in the first session and during more difficult tasks was suggested to reflect mediating strategies. However, further empirical studies are needed to evaluate the suggested functional difference between the positive and negative components of the ERP related to simple multiplication problems.

\section{Acknowledgements}

Research was supported by the Deutsche Forschungsgemeinschaft (Sonderforschungsbereich 307).

\section{References}

[1] Anderson, J.A., Spoehr, K.T, and Bennett, D.J., A study in numerical perversity: Teaching arithmetic to a neural network. In D.S. Levine and M. Aparicio (Eds.), Neural Networks for Knowledge Representation and Interference, Lawrence Erlbaum, Hillsdale, NJ, in press.

[2] Baroody, A.J., $\Lambda$ reexamination of mental arithemetic models and data: A reply to Asheraft, Der: Rer., 3 (1984) 225-230.

[3] Birbaumer. N., Elbert, T., Lutzenberger, W., Rockstroh, B. and Schwarz, J., EEG and slow cortical potentials in anticipation of mental tasks with different hemisperic involvement, Biol. Psychol., 13 (1981) 251-260.

[4] Birbaumer, N., Elbert, T., Canavan, A. and Rockstroh, B.. Slow potentials of the ecrebral cortex and behavior, Physiol. Ret., 70 (1990) $1-41$.

[5] Bourne, L.E.J. and Rickard, T.C., Mental Calculation: The Derelopment of a Cognitice Skill, Paper presented at the Interamerican Congress of Psychology, San Jose, Costa Rica, 1991.

[6] Campbel1, J.I.D. and Graham, D.J., Mental multiplication skill: Structure, process, and acquisition, Can. J. Psychol, 39 (1985) $338-366$.
[7] Campbell, J.I.D. and Oliphant, M., Representation and retrieval of arithmetic facts: A network interference model and simulation. In J.I.D. Campbell (Ed.), The Nature and Origins of Mathematical Skills, Elsevier/North Holland, Amsterdam, in press.

[8] Campbell, L.I.D., Conditions of error priming in number fact retrieval, Memory Cogn., 19 (1991)197-209.

[9] Chwilla, D.J. and Brunia, C.H.M., Effects of emotion on eventrelated potentials in an arithmetic task, J. Psychophysiol., 6 (1992) $321-332$.

[10] Coles, M.G.H., Modern mind-brain reading: psychophysiology, physiology, and cognition, Psychophysiology, 26 (1989) 251-269.

[11] Donchin, E. and Coles, M.G.H., Is the P300 component a manifestation of context updating?, Behat. Brain Sci. II (1988) $372-375$.

$\lceil 12\rceil$ Elbert. T., Lutzenberger. W., Rockstroh. B. and Birbaumer, N., Removal of ocular artifacts from the EEG - a biophysical approach to the EEG. Electroencephalogr. Clin. Nearophysiol., 60) (1985) 455-463

[1.3] Fendrich, D.W., Healy, A.F. and Bourne, L.E.Jr., Mental arithmetic: Training and retention of multiplication skill. In I. Izawa (Ed.), Applicd Cognitice Psychology: Application of Cognitice Theories and Concepts. Erlbaum, New York, in press.

[14] Inouye, T., Shinosaki. K.. Iyama, $\Lambda$. and Matsumoto, $Y .$, Localization of activated areas and directional EEG patterns during mental arithmetic, Electroencephalogr. Clin. Neurophysiol., 86 (199.3) 224-230.

[15] Kolb, B. and Whishaw, I.Q., Fundamentals of Human Neuropsychology, 3rd edn., Freeman, New York. 1990.

[16] Levin, H.S. and Spiers, P.A., Acalculia. In K.M. Heilman and E. Valenstein (Eds.), Clinical Neuropsychology, Oxford University Press, New York, 1985.

[17] Libet, B., Gleason, C., Wright, E. and Pearl, D., Time of conscious intention to act in relation to onset of cerebral activity (readiness potential), Brain. 106 (1983) 623-642.

[18] Libet, B., Unconscious cerebral initiative and the role of conscious will in voluntary action, Behat. Brain Sci, 8 (1985) $529-566$.

[19] Logan, G.D.. Towards an instance theory of automatization. Psichol. Rer., 95 (1988) 492-527.

[20] Luria, A., The frontal lobes and the regulation of behavior. In K. Pribram and A. Luria (Eds.), Psychophysiology of the Frontal Lohes, Academic Press, New York, 1973.

[21] Mayes, A.R., Human Organic Memon Disorders. Cambridge University Press, Cambridge. 1988.

[22] McCloskey, M., Caramazza, A. and Basili, A., Cognitive mechanisms in number processing and calculation: evidence from dyscalculia, Brain Cogn., 4 (1985) 171-196.

[23] McCloskey, M. and. Lindemann, A.M., MATHNET: preliminary results from a distributed model of arithmetic fact retrieval. In J.1.D. Campbell (Ed.), The Origins of Mathematical Skills, Fisevier/North Holland. Amsterdam. in press.

[24] Moscovitch, M.. and Winocur, G., The neuropsychology of memory and aging. In F.I. (raik and T.A. Salthouse (Eds.), The Handhook of Aging and Cognition. Erlbaum. Hillsdale, NJ, 1992. pp. $315-372$.

[25] Newell, $A$, and Rosenbloom, P.S., Mechanisms of skill acquisilion and the law of practice. In J.R. Anderson (Ed.), Cognitite Skills and their Acquisition. Erlbaum, Hillsdale, NJ, 1981.

[26] Oldficld, R., The assessment and analysis of handedness, Netropsychologia. 9 (1971) 97-113.

[27] Rickard, T.C., Bending the power law: a quantitative model of the transition from algorithm to association, Dissertation Proposal. University of Colorado, Boulder. $\mathrm{CO}$, unpublished.

[28] Rickard, T.C. and Bourne, I..F. Jr., An Interactive Actiration Model of Arithmetic Fact Retrietal, Institute of Cognitive Science Technical Report. University of Colorado, Boulder, CO, 1992. 
[29] Rickard, T.C. Healy, A.F. and Bourne, L.E. Jr.. On the representation of arithmetic facts: operand order, symbol, and operation transfer effects. J. Exp. Psychol.: Learning, Memory. Cogn., in press.

[30] Rockstroh, B., Elbert, T., Canavan, A., Lutzenberger, W. and Birbaumer, N., Slow Cortical Potentials and Behatiour, 2nd edn., Urban and Schwarzenberg, Baltimore, 1989.

[31] Roland, P.E. and Friberg, L., Localization of cortical areas activated by thinking, J. Neurophysiol, 53 (1985) 1219-1243.

[32] Rösler, F. and Heil. M., Toward a functional categorization of low waves: Taking into account past and future events, Psy(hophysiology, 28 (1991) 344-364.

[33] Rösler. F., Schumacher, G. and Sojka, B., What the brain reveals when it thinks: Event-related potentials during mental rotation and mental arithmetic, German J. Psychol., 14 (1990) $185-203$.
[34] Ruchkin, D.S., Johnson, R., Mahaffey, D. and Sutton, S., Toward a functional categorization of slow waves, Psychophysiology, 25 (1988) 339-353.

[35] Ruchkin, D.S., Johnson, R., Canoune, H. and Ritter, W., Event-related potentials during arithmetic and mental rotation, Electroencephalogr. Clin. Neurophysiol., 79 (1991) 473-487.

[36] Siegler. R.S., Startegy choice procedures and the development of multiplication skill, J. Exp. Psychol.: General. 117 (1988) $258-275$.

[37] Sokol, S.M., McCloskey, M., Cohen, N.J. and Aliminosa, D., Cognitive representations and processes in arithmetic: evidence from the performance of brain-damaged patients, J. Exp. Psychol.: Learning, Memory, Cogn., 8 (1991) 320-335.

[38] Stazyk, E.H., Ashcraft, M.H. and Hamann, M.S., A network approach to mental multiplication. J. Exp. Psychol.: Learning, Memory, Cogn., 8 (1982) 320-335. 